\title{
Update on chronic immune thrombocytopenic purpura (ITP) Robert McMillan
}

\author{
Address: The Scripps Research Institute, 10666 North Torrey Pines Road, La Jolla, CA 92037, USA \\ Email: Robert McMillan - robtmcmillan@sbcglobal.net
}

from Current trends in leukemia, lymphoma and myeloma

White Plains, NY, USA. 31 January 2009

Published: 26 June 2009

Journal of Hematology \& Oncology 2009, 2(SuppI I):A5 doi:I0.1 I86/I756-8722-2-SI-A5

This abstract is available from: http://www.jhoonline.org/content/2/SI/A5

(c) 2009 McMillan; licensee BioMed Central Ltd.

\section{Introduction}

Chronic ITP is an autoimmune disorder manifested by immune-mediated platelet destruction and suppressed platelet production. The following article will discuss: normal platelet production and circulation; ITP pathophysiology; and diagnosis and treatment options for ITP.

\section{Platelet production and circulation}

Thrombopoietin (TPO), the major cytokine affecting all phases of platelet production, is produced primarily by the liver and binds to the c-Mpl receptor located on platelets, stem cells and megakaryocytes. Upon stimulation, pluripotential stem cells produce cells committed to megakaryocyte differentiation. These cells initially undergo mitosis followed eventually by endomitosis resulting in a progressive increase in size with cells ranging from $2 \mathrm{~N}$ to $64 \mathrm{~N}$ and a proportional cytoplasmic increase. Platelet formation occurs in the cytoplasm and the number of platelets produced/megakaryocyte is roughly proportional to megakaryocyte size. Platelet production is regulated as follows: the liver produces a constant quantity of TPO each day and a large portion of this binds to circulating platelets; unbound TPO is available to stimulate platelet production. In thrombocytopenic states due to reduced platelet production, less TPO is adsorbed by circulating platelets and more is available to stimulate platelet production. In ITP, TPO levels are normal or slightly increased probably due to TPO binding to platelets which are then destroyed, removing TPO from the circulation.

Circulating platelets have an intravascular survival of 710 days. About $30 \%$ are located in an exchangeable splenic pool which, if enlarged, may result in peripheral thrombocytopenia (e.g., patients with congestive splenomegaly). Platelets are removed from the circulation due to utilization in maintaining vascular integrity or because of old age.

\section{Differential diagnosis of thrombocytopenia}

Thrombocytopenia can result from: decreased platelet production, platelet redistribution (enlarged splenic pool) or platelet destruction. Decreased production may be due to myeloproliferative disorders (leukemia, myeloma, etc.), myelodysplasia, aplasia or hypoplasia, drugs (chemotherapy, alcohol, etc.), malignant infiltration or may be inherited. Decreased production is usually diagnosed by evaluation of the blood count and bone marrow. Abnormal platelet distribution involves an enlarged spleen and is most commonly due to congestive splenomegaly although other disorders such as lymphoma, Gaucher's disease, etc. may also have some element of redistribution. There are multiple causes of platelet destruction including infection, thrombotic thrombocytopenic purpura, disseminated intravascular coagulation or immune causes due to alloantibodies, drug-dependent antibodies, acute ITP or chronic ITP or ITP-like syndromes associated with collagen vascular or lymphoproliferative disorders.

Chronic ITP is a diagnosis of exclusion. The classic ITP patient presents with petechiae and easy bruising with or without mucosal bleeding. The blood count shows only isolated thrombocytopenia with scattered large platelets. Anemia is absent, unless there is bleeding or immune hemolysis. The bone marrow is normal except for an increase in megakaryocytes. 


\section{Pathogenesis of chronic ITP}

Thrombocytopenia in ITP may be due to platelet destruction, suppressed platelet production or both. Evidence for platelet destruction: (1) infusion of ITP plasma into normal recipients may cause thrombocytopenia; (2) intravascular platelet survival is reduced; (3) autoantibodyinduced platelet phagocytosis can be demonstrated and (4) ITP cytotoxic T cells can lyse autologous platelets and (5) many patients respond to therapies which affect platelet destruction, such as splenectomy, intravenous gammaglobulin (IVIg), anti-D, etc. Evidence for suppressed platelet production: (1) platelet turnover (a measure of platelet production) is either normal or reduced in over $80 \%$ of ITP patients rather than increased as would be expected if platelet destruction were the only mechanism; (2) damage to ITP megakaryocytes can be demonstrated morphologically; (3) autoantibody from some ITP patients suppresses platelet production and maturation in vitro; and (4) the majority of patients respond to TPO mimetics which stimulate platelet production. It is likely that ITP cytotoxic T cells also damage megakaryocytes, since they have similar surface proteins, but this has not been confirmed.

\section{Thrombopoietin mimetics: a new therapeutic approach}

A few years ago, thrombopoietin and agents, containing the active portion of thrombopoietin, were developed and used to treat ITP patients. However, their use was discontinued when antibodies against these agents developed which rendered them inactive and, in addition, affected the function of the recipient's normal thrombopoietin. Subsequently, a variety of agents have been developed, including small peptide or non-peptide molecules, which have no sequence homology with TPO but are able to stimulate platelet production. Two of these agents, romiplostim (NPlate ${ }^{\varpi}$ ) and eltrombopag (Promacta ${ }^{\varpi}$ ) have undergone clinical trials and recently received FDA approval for use in chronic ITP.

Based on these clinical trials, it has been shown that both agents increase the platelet count in most chronic ITP patients most of the time resulting in reduced bleeding and allowing many patients to reduce or stop other ITP therapies. Although adverse events have been infrequent, there are potentially serious adverse events which may occur with long-term use of these agents: (1) severe thrombocytopenia upon stopping the drug with platelet counts significantly below pre-treatment levels for up to two weeks requiring, in some cases, rescue therapy; (2) increased bone marrow reticulum which thus far has been reversible in the few patients studied; (3) possible stimulation of leukemic cell growth (11 of 44 patients with myelodysplasia, receiving romiplostim, developed disease progression, four progressing to acute granulocytic leukemia); (4) hepatobiliary toxicity (eltrombopag). Potentially serious adverse events which have not been seen thus far include: thrombosis/thromboembolism, antibody formation against the drug and stem cell depletion.

\section{Therapy of chronic ITP \\ Emergency therapy}

Emergency therapy should be considered if the platelet count is $<5000$ per $\mu \mathrm{l}$ or if there is significant mucosal or CNS bleeding. Hospitalization may be indicated. Patients should receive intravenous gammaglobulin (IVIG) $1.0 \mathrm{~g} /$ $\mathrm{kg} / \mathrm{d}$ IV for two days and/or methylprednisolone $1.0 \mathrm{~g} / \mathrm{d}$ IV for 3 days. Platelet transfusion may be indicated to control bleeding.

\section{First-line therapy}

A percentage of ITP patients will attain a spontaneous partial or complete remission if their platelet count can be maintained until this occurs. The purpose of first-line therapy is to maintain a safe platelet count $(>25-30,000$ per ul) to either provide time for spontaneous recovery or to give therapy which will reverse the abnormal immune response. If no response occurs after therapy for several weeks or if patients relapse after a response, second line therapy should be given. The following therapies should be considered: (1) prednisone - $1 \mathrm{mg} / \mathrm{kg}$ daily p.o. until a response occurs followed by tapering over a period of several weeks; (2) high-dose dexamethasone $-40 \mathrm{mg}$ PO or $\mathrm{IV} / \mathrm{d} \times 4$ every 14 days $\times 4$; (3) Anti-D $-50-75 \mathrm{ug} / \mathrm{kg}$ IV prn to maintain the platelet count $>30,000$ per ul; (4) rituximab $-375 \mathrm{mg} / \mathrm{M}^{2} \mathrm{IV}$ per week $\times 4$; or (5) thrombopoietin mimetics - romiplostim (1-10 ug/kg s.c. weekly) or eltrombopag (25-75 mg po daily on an empty stomach) aiming to maintain a platelet count of $\geq 50,000$ per ul.

\section{Second line therapy}

If there is continued, symptomatic thrombocytopenia after therapeutic trials of the above agents, there are two possible considerations: (1) splenectomy or (2) continue the thrombopoietin mimetic, if the patient continues to respond. Splenectomy would seem to be the wiser choice at this time since long-term results are not yet available for the thrombopoietin mimetics.

Splenectomy has been used for chronic ITP for over 50 years and there are solid long-term data showing that about two thirds of patients are cured of their disease. Immunizations for pneumococcus, meningococcus and $H$. influenza should be given and, if possible, the platelet count should be raised above 50,000 per ul pre-op using corticosteroids, IVIG or anti-D. Platelets should be available but not given unless excessive bleeding occurs. Laparoscopic splenectomy is recommended since results are 
equal to standard splenectomy and patients recover more rapidly and require shorter hospitalizations.

\section{Therapy of refractory ITP}

For patients failing splenectomy, several options are available. A retrial of prednisone \pm dapsone or danazol, rituximab or one of the TPO mimetics should be considered. Patients, who do not respond, can be treated with chemotherapeutic agents (azathioprine or oral cyclophosphamide) or immunosuppressants (cyclosporine or mycophenolate mofetil). In patients, who remain thrombocytopenic and have symptomatic bleeding, consideration may be given to more toxic therapy such as high-dose cyclophosphamide, combination chemotherapy or stem cell transplantation.

Publish with Bio Med Central and every scientist can read your work free of charge

"BioMed Central will be the most significant development for disseminating the results of biomedical research in our lifetime. " Sir Paul Nurse, Cancer Research UK

Your research papers will be:

- available free of charge to the entire biomedical community

- peer reviewed and published immediately upon acceptance

- cited in PubMed and archived on PubMed Central

- yours - you keep the copyright

Submit your manuscript here:

http://www.biomedcentral.com/info/publishing_adv.asp 